\title{
La médiation du patrimoine en ligne : réflexion autour d'une cyber-exposition pour le centenaire du génocide arménien au Québec
}

\section{Marie-Blanche Fourcade}

\section{(2) OpenEdition}

\section{Édition électronique}

URL : https://journals.openedition.org/ocim/1602

DOI : 10.4000/ocim. 1602

ISSN : 2108-646X

Éditeur

OCIM

\section{Édition imprimée}

Date de publication : 1 novembre 2015

Pagination : 47-50

ISSN : 0994-1908

\section{Référence électronique}

Marie-Blanche Fourcade, «La médiation du patrimoine en ligne : réflexion autour d'une cyber-

exposition pour le centenaire du génocide arménien au Québec », La Lettre de I'OCIM [En ligne], 162 I

2015, mis en ligne le 01 novembre 2016, consulté le 08 décembre 2022. URL : http://

journals.openedition.org/ocim/1602 ; DOI : https://doi.org/10.4000/ocim.1602

Ce document a été généré automatiquement le 29 septembre 2020

Tous droits réservés 
La médiation du patrimoine en ligne : réflexion autour d'une cyberexposition pour le centenaire du génocide arménien au Québec

Marie-Blanche Fourcade 
Tournage des capsules vidéos durant la collecte patrimoniale à l'École arménienne Sourp Hagop, Montréal, avril 2015

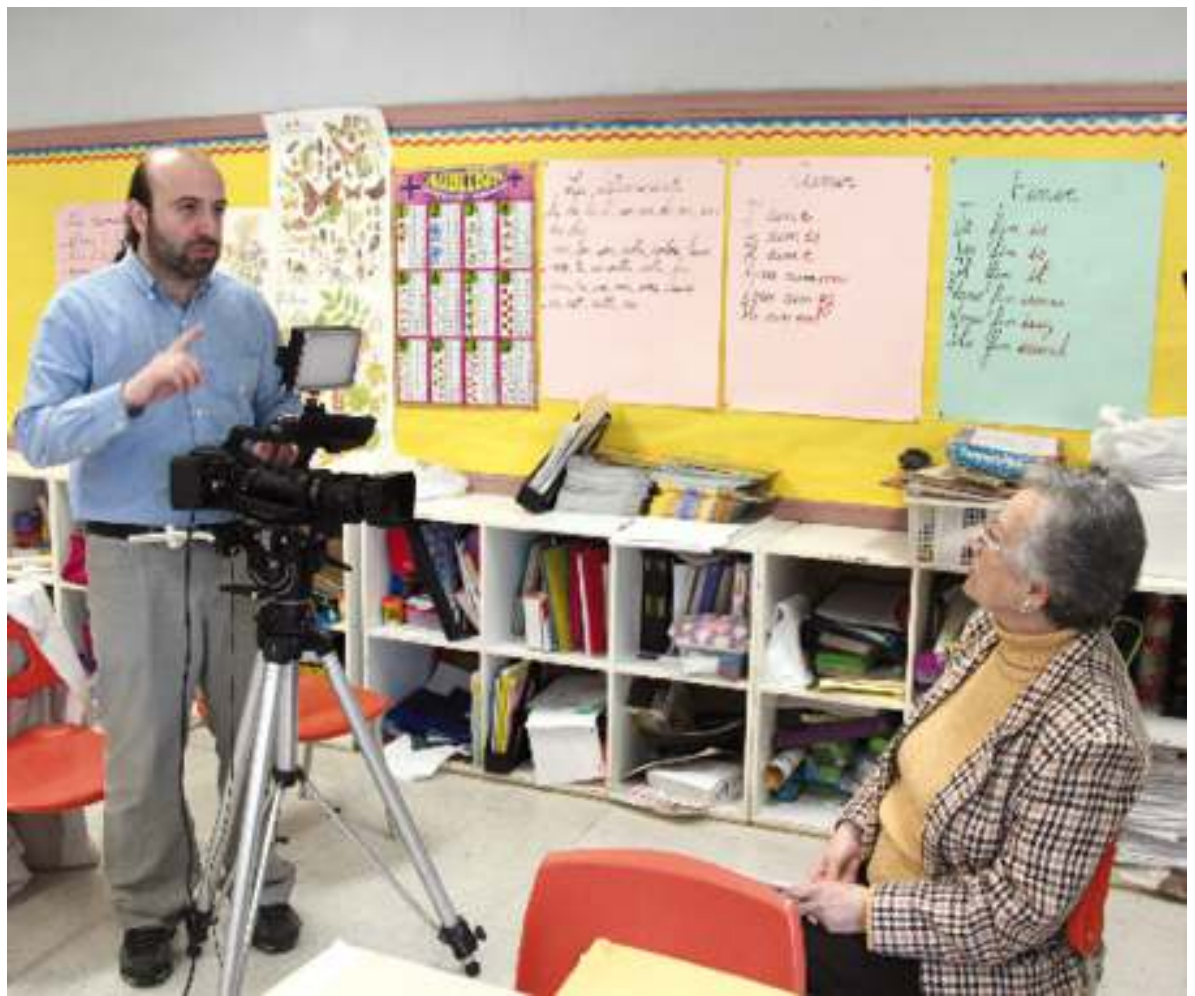

(c) M.-B. Fourcade

1 L'année 2015 est une année charnière pour la nation arménienne et ses communautés dispersées à travers le monde. Année de commémoration du centenaire du génocide arménien, il s'agit d'un temps privilégié pour la production d'évènements qui donnent l'occasion de faire connaître au grand public l'histoire et la culture arméniennes, mais qui rendent également visibles les enjeux politiques et humains associés à la reconnaissance du génocide arménien. Dans ce contexte, le Comité canadien du centenaire du génocide des Arméniens ${ }^{1}$ a choisi plusieurs supports de médiation, logés à l'enseigne de conférences, de productions artistiques et d'exposition. Parmi ces multiples projets, une cyber exposition dédiée au patrimoine de la diaspora, et plus particulièrement à celui qui évoque la période pré-génocidaire et génocidaire, a été mise en chantier pour être lancée au printemps 2016. La démarche faisait écho à ma recherche doctorale sur le patrimoine familial de la diaspora arménienne au Québec ${ }^{2}$ et à plusieurs recommandations émises, en conclusion du travail ethnographique, sur la conservation et le partage du patrimoine arménien comme outil de valorisation de la culture et de l'histoire des communautés dispersées.

Depuis juin 2014, un comité de membres de la communauté arménienne de Montréal que je co-anime participe à une réflexion sur ce qui doit être présenté et comment. Au cours de la préparation du projet, il a fallu, entre autres, mener un travail exploratoire sur le Web afin de saisir ce qui était déjà mis en place en termes de produits numériques : de quelle manière le sujet avait-il été abordé et quels étaient les objectifs des concepteurs. Parmi les réalisations présentes en ligne, je me suis plus particulièrement penchée sur des plateformes dont la démarche s'appuyait, en amont, sur la participation d'acteurs ou de 
témoins-clés. C'est ainsi, en croisant ces recherches et le projet en cours de réalisation que plusieurs questions ont émergé sur le rôle de la médiation en ligne du patrimoine et plus spécifiquement dans le cas de la communauté arménienne, notamment à ce qui a trait à la création d'un espace privilégié de partage. Après une évocation de la démarche qui mène à la production de la cyber exposition et l'exploration de deux productions en ligne qui ont largement alimenté notre réflexion collective au sein de l'équipe, je proposerais sous forme de pistes d'exploration plusieurs contributions possibles du numérique dans la médiation du patrimoine arménien.

\section{De la maison à l'espace numérique : collecter les traces du génocide arménien à Montréal}

3 Avant même de se pencher sur la réalisation du projet, il me faut revenir aux éléments structurants qui définissent le mandat de l'exposition en ligne. En premier lieu, il était impératif pour notre équipe que l'exposition fasse sortir de la sphère familiale, des trésors de culture matérielle et de documentation qui évoquent à la fois une histoire collective et des histoires individuelles. Ces histoires, faites de ruptures, de traumatismes, d'oublis et de déplacements, sont finalement sous-représentées dans le présent puisque peu d'objets ont subsisté et que les récits restent dans de nombreux cas dans l'enceinte de la famille. Dans le contexte de rareté de la culture matérielle, il est donc essentiel de non seulement prendre conscience collectivement de leur existence, car ils sont pour la plupart méconnus du grand public et des Arméniens eux-mêmes, mais également d'initier une démarche de responsabilisation à leur égard qui vise avant tout de bonnes pratiques de conservation et de valorisation. En deuxième lieu, la démarche expographique devait s'appuyer sur l'histoire des objets personnels qui contribuent à de grands récits familiaux qui permettent tout autant de valoriser des trajectoires d'objets que des biographies porteuses de mémoires. En dernier lieu, il fallait que la démarche de collecte de matière pour l'exposition se réalise dans un processus de co-construction avec les membres de la communauté arménienne à l'échelle du Québec, au moins, et qu'elle puisse toucher plusieurs générations de participants. 


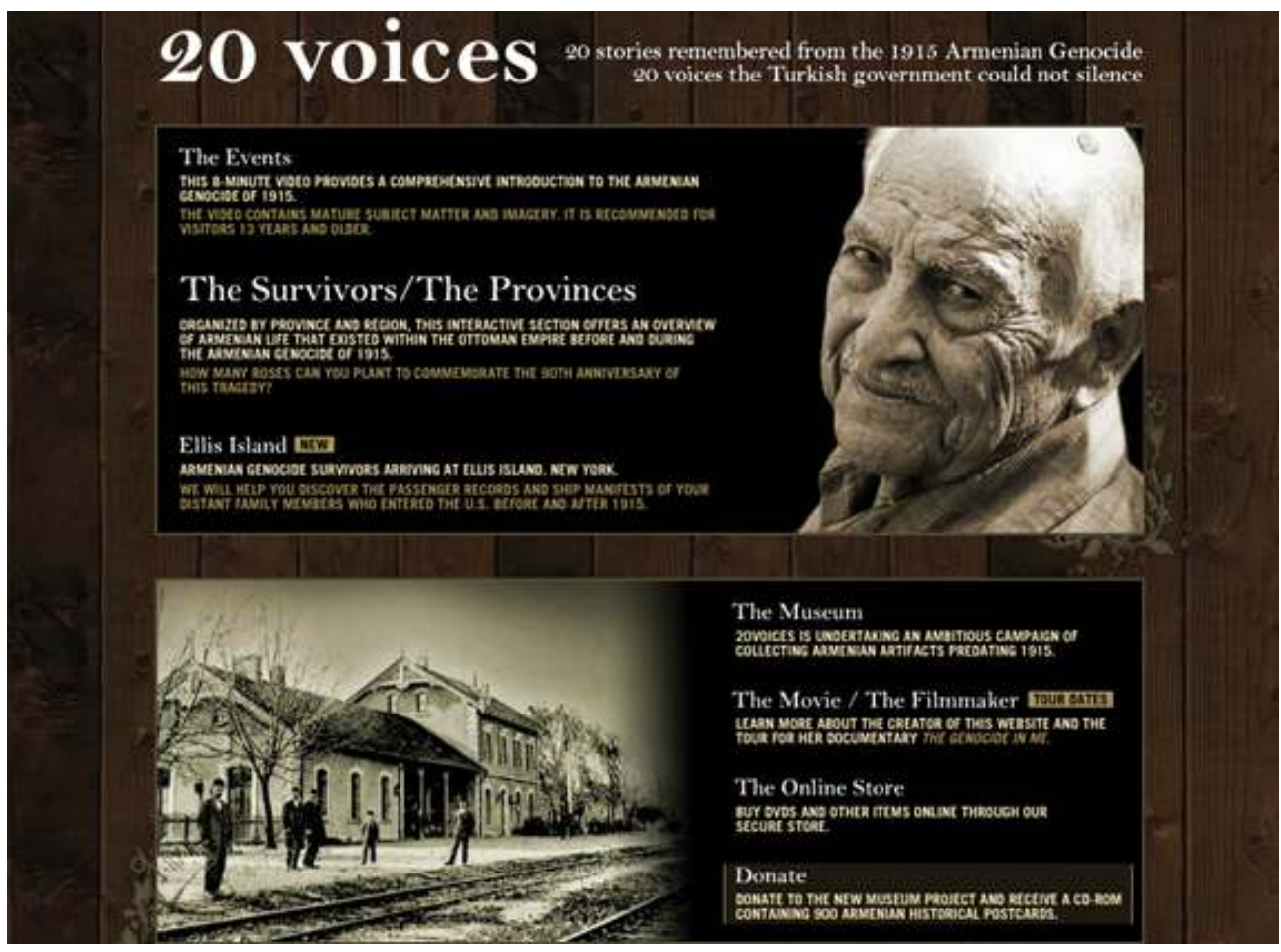

4 En suivant les principes fixés, une campagne de collecte numérique d'artéfacts et de documents a été menée au printemps 2015 à Montréal. Pensée comme un évènement extraordinaire dans le temps communautaire, cette collecte s'inspire du travail des cliniques de mémoires développé depuis 2003 par le Centre d'histoire de Montréal ${ }^{3}$. L'invitation est simple : venir faire un don de mémoire comme certains feraient un don du sang afin de pouvoir alimenter l'histoire de sa communauté, qu'elle soit culturelle, résidentielle ou professionnelle. Ainsi, à l'appel lancé par l'équipe dans les médias sociaux, la télévision et la presse, des membres de la communauté principalement installée à Montréal sont venus faire don des histoires de leurs objets, témoins de la culture arménienne avant les grandes migrations causées par le génocide de 1915. D'abord enregistrés et décrits pour entrer dans l'inventaire de la collection numérique, les objets sont ensuite présentés par leur propriétaires ou leur dépositaires sous l'objectif de la caméra. En complément de cette démarche publique, la collecte a aussi été menée dans plusieurs écoles arméniennes, s'inspirant cette fois, d'une autre initiative du Centre d'histoire de Montréal : "Vous faites partie de l'histoire! » qui propose à des élèves d'explorer leur patrimoine familial et de partager l'une de leurs trouvailles en classe. Selon un même esprit, plus d'une quarantaine d'enfants de différents âges de l'école primaire à Montréal et à Toronto ont accepté de rechercher des objets associés au génocide arménien et de constituer un court dossier de recherche qui faciliterait l'intégration à l'inventaire. À ce jour, la collecte a permis de récolter et de documenter environ 130 objets d'intérêt susceptibles de nourrir l'expographie. Une base de données multimédia est en cours de constitution et la conception de l'exposition sera amorcée à partir de janvier 2016. 


\section{La mise en ligne du patrimoine et des mémoires : deux exemples arméniens}

5 Deux projets de conservation de la mémoire arménienne en ligne ont plus particulièrement alimenté le cheminement de notre projet. Le premier d'entre eux, 20 voices $^{5}$, a été réalisé en 2004 à Montréal par la documentariste, Araz Artinian qui, dans sa volonté de réaliser un documentaire éducatif sur le génocide, a collecté des témoignages de survivants sur la côte est nord-américaine, au Canada et aux États-Unis. Outre leur contribution à la réalisation du film The Genocide in me, les témoignages sont présentés sur une plateforme Web. L'articulation des récits s'appuie sur une expérience de voyage, ou plutôt sur une démarche commémorative qui mène dans les différentes provinces de l'Empire ottoman habitées par la population arménienne avant le génocide ${ }^{6}$. Une carte interactive facilite le survol des provinces à visiter et chacune d'entre elles, permet d'écouter le témoignage d'un survivant. Des documents d'archives, des photographies et des extraits musicaux bonifient l'écoute. La présence de la plateforme sur le Web a eu plusieurs effets. La présentation des témoignages a d'abord montré l'importance de « donner une voix » tel que le dira la conceptrice, aux mémoires individuelles et ce, au-delà d'un grand récit collectif. Elle a également permis de prendre conscience de la fragilité de ce patrimoine oral, compte tenu de l'âge des survivants. Ce patrimoine ne doit donc pas être seulement conservé, mais bel et bien sauvegardé. Le succès de fréquentation du site a enfin montré que la création d'un dépôt de mémoire numérique qui favorise la diffusion et le partage de témoignages, faisait également l'objet d'une grande fierté pour toute la communauté.

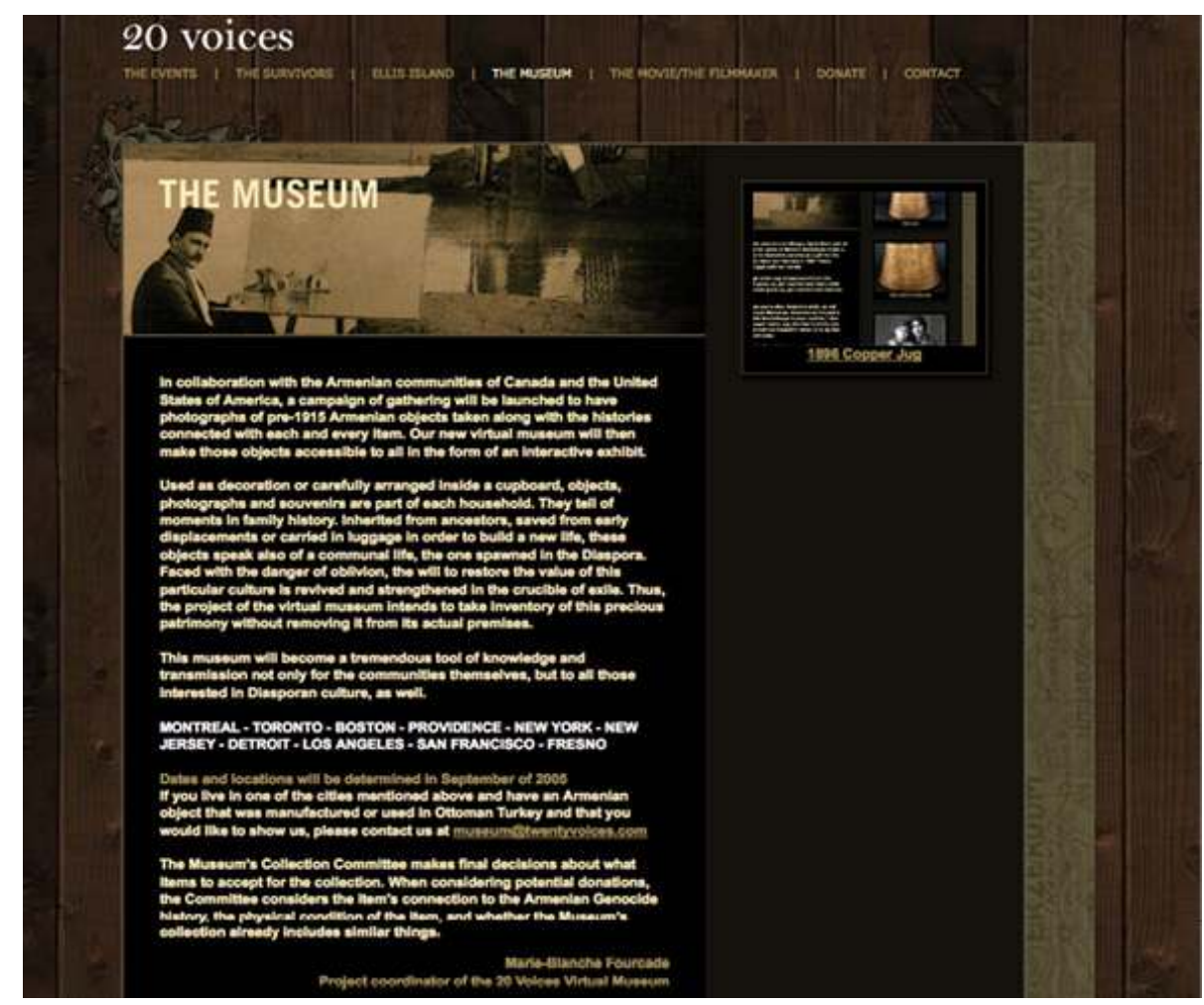


Houshamadyan, le second projet, est développé depuis 2010 par une équipe internationale de chercheurs et spécialistes en études arméniennes dont le siège est à Berlin. Dans la lignée de 20 voices, la mission principale de l'organisation éponyme est de « reconstruire et préserver la mémoire de la vie arménienne dans l'Empire ottoman ${ }^{7}$. Plus que le rassemblement et la conservation d'archives documentaires et multimédias concernant les villes et les villages ottomans, l'objectif scientifique est de compléter un corpus de références jusqu'à présent lacunaire en ce qui concerne la présence arménienne dans l'Empire ottoman et, ainsi, rétablir ou donner une place plus importante à l'historiographie arménienne. Le terme Houshamadyan signifie littéralement « livre de mémoire », ce qui permet de saisir le travail de réécriture précédé du rassemblement et de la mise en ordre du passé dans une perspective de reconstruction, mais surtout de transmission. Afin de renforcer l'action de la collecte à long terme, la plateforme possède également une dimension participative, puisque les visiteurs sont invités à envoyer en format numérique tout document - revues, correspondance, document administratif, photographies... - afin qu'il soit ajouté à la base de données et éventuellement partagé sur le Web. Le développement progressif d' Houshamadyan a, tout autant que 20 voices, influencé la réflexion des membres de l'équipe. En effet, si les motifs qui ont mené à la création de la plateforme et les effets réparateurs qu'elle semble manifester sur la mémoire constituent une part importante de l'intérêt qui lui est portée, l'envergure des résultats et la capacité de fédérer un grand nombre d'acteurs agit également comme motivateur dans la création d'une cyber exposition.

Page d'accueil du site Houshamadyan, novembre 2015

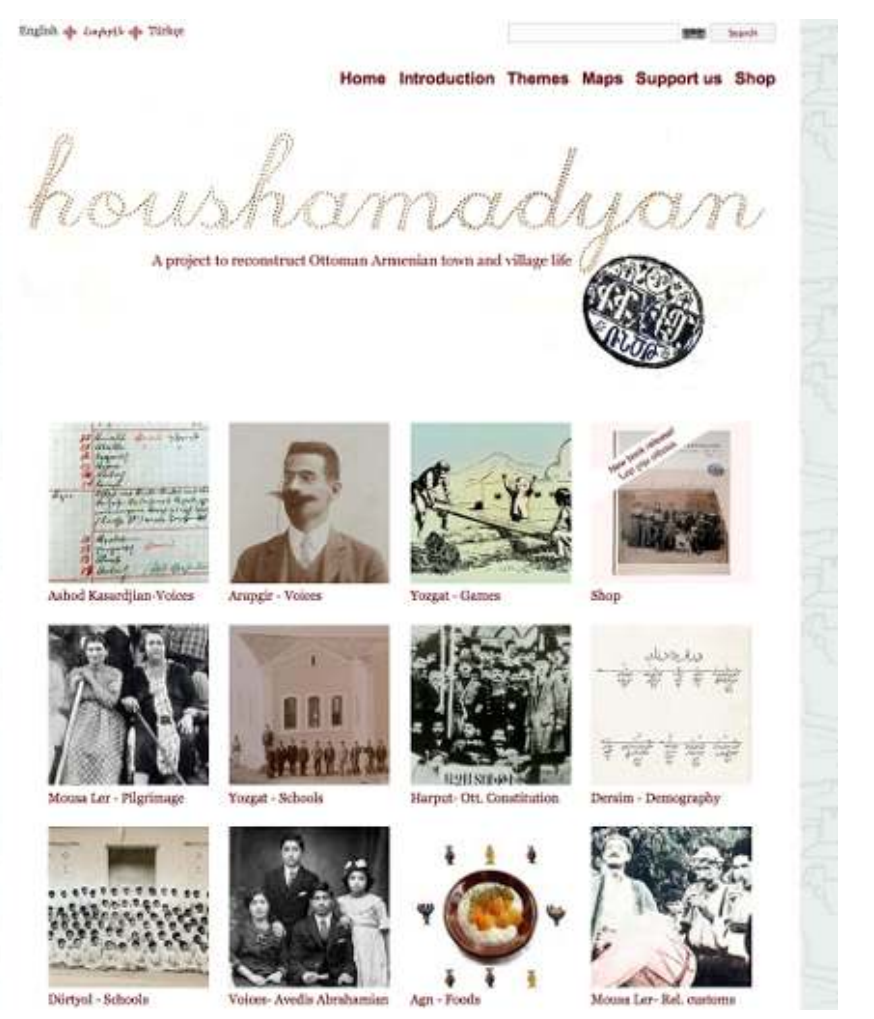




\section{Penser la contribution du numérique dans la médiation du patrimoine}

7 À partir de l'expérience en cours et des projets évoqués, l'une des pistes de réflexion qui s'impose d'emblée dans l'analyse des démarches de valorisation en ligne s'inscrit à l'enseigne du rassemblement de ce qui est dispersé. Cette idée de contrer la dispersion est d'autant plus forte qu'elle s'inscrit dans un contexte de mobilité diasporique. Autrement dit, le numérique offre la possibilité de mettre en commun, et cela, sans égard aux distances, aux frontières et aux diverses contingences physiques si ce n'est celle d'un accès au Web, un ensemble d'objets et de récits qui n'ont pas nécessairement de liens de sens ou de contacts physiques entre eux. Dans le cas des objets du patrimoine arménien, s'ils participent à une histoire commune, ils témoignent tous d'histoires de famille variées et sont conservés dans des foyers qui ne permettent pas leur cohabitation. La convergence d'empreintes d'objets et d'enregistrement de récits vers un même espace d'exposition offre ainsi non seulement une contrepartie à la dispersion, mais permet aussi de produire un dialogue renouvelé sur le patrimoine diasporique.

Par le fait du rassemblement, une autre piste de réflexion s'annonce, celle de la création d'un espace de conservation alternatif inclusif et autonome qui rend visible une collection jusqu'alors bien peu montrée en raison de sa nature intime. Cet espace prend le rôle d'intercesseur avec le passé qui permet ainsi de montrer les preuves à échelle humaine, ou pourrait-on dire micro, des évènements traumatiques qui ont eu lieu. Non plus seulement le reflet et la référence d'une communauté qui revendique la reconnaissance du premier génocide du $\mathrm{XX}^{\mathrm{e}}$ siècle, tâchant coûte que coûte de transmettre son histoire d'une génération à l'autre, l'exposition en ligne devient également le reflet d'un public plus large, délocalisé. Il favorise de la sorte la création d'une communauté d'intérêts et pratiques qui réunit participants, visiteurs, curieux et amateurs, qu'ils soient arméniens, d'origine arménienne, ou qu'ils aient un intérêt pour l'Arménie, le patrimoine, les histoires de vie voire de simples curieux de la culture en général.

\section{Pour conclure}

L'analyse du double travail d'accompagnement que je mène auprès du comité qui s'est engagé dans la collecte patrimoniale en dialogue, et d'exploration des productions culturelles en ligne que je poursuis dans le cadre de mes recherches, me laisse croire que le numérique permet d'agir à la marge lorsque l'institution muséale n'est pas en mesure d'intervenir. Dans le cas du patrimoine arménien où la légitimité d'un musée diasporique installé à Montréal a du mal à se faire valoir, que ce soit pour des questions de collections, de localisation et de moyens financiers, le Web s'impose comme un support qui permet la transgression des impossibles. Parmi ceux-ci, on peut compter celui de partager sans retirer aux propriétaires le bénéfice de leurs souvenirs; celui d'acquérir des objets sans discrimination de valeur d'art, d'unicité ou d'authenticité, mais plutôt sur la base de l'expérience et des émotions contenues; celui également de rendre accessible sans imposer de présence physique; celui enfin d'avoir un musée sans pâtir d'un poids économique. Plus encore le numérique offre une occasion aux membres d'une communauté de prendre la responsabilité de faire collection à partir de ses traces du passé, de ses expériences sensibles et des souvenirs dessinant ainsi les contours d'une 
communauté choisie qui ne se limite plus seulement aux liens de sang mais s'ouvre également aux liens de cœur et d'esprit.

\section{NOTES}

1. Comité canadien du centenaire du génocide des Arméniens, http://genocidecentennial.ca, page consultée le 31 octobre 2015.

2. Fourcade, M.-B. Habiter l'Arménie au Québec. Ethnographie d'un patrimoine en diaspora. Québec : Presses de l'Université du Québec, 2011.

3. Leclerc, J.-F. et Pirès, J. Du patrimoine à la construction d'une identité urbaine plurielle: le rôle d'un équipement culturel municipal, le Centre d'histoire de Montréal. Office de consultation publique de Montréal, 2004, http://ocpm.qc.ca/sites/import.ocpm.aegirvps.net/files/pdf/PD04/3g.pdf, page consultée le 31 octobre 2015 ; Leclerc, J.-F. Des cliniques de mémoire pour enrichir notre patrimoine commun, Nos diverses cités, ${ }^{\circ} 7,2010$, pp. 104-109.

4. "Vous faites partie de l'histoire !" http://ville.montreal.qc.ca/vousfaitespartiedelhistoire/, page consultée le 31 octobre 2015.

5. 20 voices, http://20voices.com/, page consultée le 31 octobre 2015

6. Fourcade, M.-B. Twentyvoices.com : un autre regard sur la mémoire du génocide arménien, Ethnologie française, numéro thématique : "Mémoires plurielles, mémoires en conflit", vol. 37 (3), 2007, pp. 525-531.

7. Houshamadyan, A project to reconstruct Ottoman Armenian town and village life, www.houshamadyan.org/en/home.html, page consultée le 31 octobre 2015

\section{RÉSUMÉS}

La démarche à dimension participative présentée ici met en évidence la contribution du numérique à la valorisation en ligne d'un patrimoine jusque-là dispersé et à la constitution dans la cadre d'un processus de co-construction avec les membres de la communauté arménienne au Québec - d'une base de données multimédia, véritable espace de conservation autonome et alternatif à l'institution muséale.

\section{INDEX}

Mots-clés : médiation, patrimoine 


\section{AUTEUR}

MARIE-BLANCHE FOURCADE

professeure associée au Département d'histoire de l'art de l'université du Québec à Montréal fourcade.marie-blanche@uqam.ca 